\title{
Analytical Solution of Stresses of Tapered Interference Fit
}

\author{
Peng SHANG \\ School of Equipment Engineering, Engineering University of CAPF, \\ China 710086 \\ sipone@163.com
}

\begin{abstract}
.
Tapered interference fits can avoid the influence of keyways on the parts strength and transfer large torques. In this paper, a model was developed to study the influence of the taper and the rotational speed on the interference fit between a propeller hub and a shaft. Using the classic elastic plane stress theory, the exact solutions of the radial stresses, tangential stresses of the hub and shaft are derived. Taking a screw propeller system as an example, the above solutions were calculated by using the numerical method. The results show that the taper plays a key role in the interference fit. Improving the stress distribution of the hub is an effective approach to increase the connection strength. The present analytical solutions are expected to be useful in the structure design of tapered interference fits for propeller hubs and shafts.
\end{abstract}

Keywords: analytical solution, stress, taper, interference fit

\section{Introduction}

Interference fits are widely used in gear design for fixation of pinions, couplings, and the like on shafts. They can transfer large torques, are easy to produce and offer significant cost advantages [1]. Commonly a cylindrical interference fit is applied. Such a clearance between hub and shaft is chosen, that the stresses of all regions of the fit can be calculated by Lame's solution. The design and stress analysis of cylindrical interference fits are studied broadly and delicately, most of which are concerned with a laminated composite tube which is interference fitted onto other structure like a bearing bush or a hub. The model is developed as a 
hollow cylinder is subjected to internal or external pressure, depending on the relative location in the joint assembly and the elastic solution can be found for such a case in the literature [2-9]. But the solution of cylindrical interference fits is not very suitable for screw propeller systems because the propeller hub and shaft are connected together by tapered interference fits.

\begin{tabular}{|llll|}
\hline \multicolumn{2}{|l|}{ Nomenclatures } & & \\
$E$ & elastic moduli [Gpa] & $r$ & radius [m] \\
$K$ & reciprocal of taper & $u$ & radial displacement [m] \\
$M$ & torque [N/m] & $\sigma$ & stress [Pa] \\
$N$ & rotational speed [rpm] & $\varepsilon$ & strain \\
$R$ & outer radius of propeller & $\mu$ & Poisson's ratio \\
$f$ & hub [m] & & \\
subscripts & & \\
$H$ & hub & $r$ & radial direction \\
$S$ & shaft & $S$ & small end \\
$b$ & big end & $\theta$ & tangential direction \\
\hline
\end{tabular}

Accordingly, the aim of this paper is to develop a better calculation method used in the design of propeller hubs and shafts for tapered interference fits which are affected by the taper and rotating speed. Using the classic elastic plane stress theory, the equations of the tapered interference fits are derived. Then the exact solutions of the radial stress, tangential stress and of the tapered interference fit are obtained. Finally taking the tapered interference fit of a screw propeller system as an example the above solutions are calculated and plotted.

\section{Analytical model}

In this research, the structure analyzed here is only the part of the tapered interference fit of a screw propeller system. The tapered interference fit between the propeller hub and shaft is shown in Fig. 1. The radiuses of the small end and big end of the propeller shaft are indicated as $r_{s}$ and $r_{b}$ respectively. The mean radius of the propeller shaft is represented as $r_{m}$. $R$ is the outer radius of the 
propeller hub and $L$ is the length of the tapered interference fit. Further we stipulate that the elastic moduli $E$ and Poisson's ratio $\mu$ as well as the density $\rho$ of the propeller hub and shaft are equal.
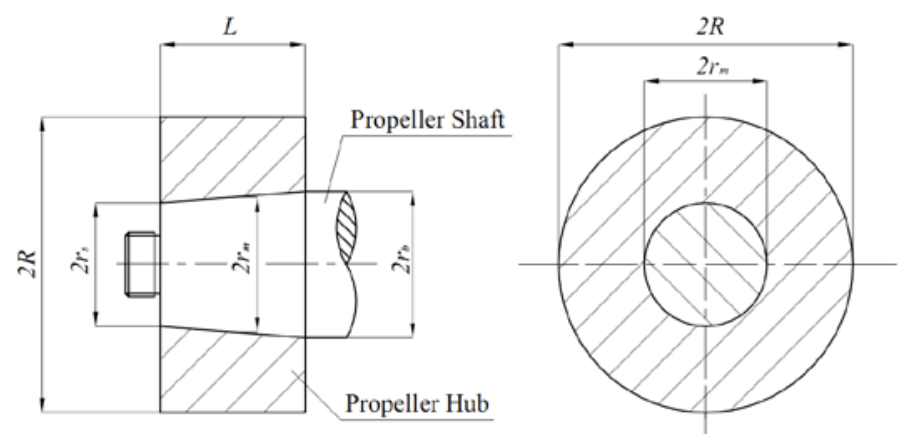

Fig. 1 The tapered interference fit between the propeller hub and shaft.

To study the connection characteristics for the tapered interference fit of the propeller hub and shaft, the influence of the taper $K$ on the stresses of the propeller hub and shaft are investigated as follow:

Using the classic elastic plane stress theory, the equilibrium differential equation can be expressed as

$$
\frac{\partial \sigma_{r}}{\partial r}+\frac{\partial \tau_{\theta r}}{r \partial \theta}+\frac{\sigma_{r}-\sigma_{\theta}}{r}+\frac{\rho r \pi^{2} N^{2}}{900}=0,
$$

and the strain-displacement relation and constitutive equation can be given as

$$
\varepsilon_{r}=\frac{d u}{d r}, \quad \varepsilon_{\theta}=\frac{u}{r},
$$

$$
\sigma_{r}=E \varepsilon_{r}+\mu \sigma_{\theta}, \quad \sigma_{\theta}=E \varepsilon_{\theta}+\mu \sigma_{r}
$$

Suppose $p$ is the contact pressure of the tapered interference fit, and then the boundary conditions are given in Eq. (4) in order to guarantee the equations above have a unique solution.

$$
\left.\sigma_{r, H}\right|_{r=r_{m}}=\left.\sigma_{r, S}\right|_{r=r_{m}}=-p,\left.\quad \sigma_{r, H}\right|_{r=R}=0,
$$

where $p$ can be given as

$$
M=0.5 \pi L f p\left(r_{s}+r_{b}\right)^{2} .
$$


The taper of the propeller shaft can be expressed as $1: K$, and the length of the tapered interference fit $L$ can be derived as

$$
L=K\left(r_{b}-r_{s}\right) .
$$

The propeller shaft is a solid shaft, so the radial stress is a finite value for $r=0$. Then combine Eqs. 1-6, the radial stresses and tangential stresses of the propeller hub and shaft deduce to Eqs. 7-10 respectively,

$$
\begin{gathered}
\sigma_{r, H}=-\frac{2 M\left(R^{2}-r^{2}\right)}{\pi f K\left(r_{b}-r_{s}\right)\left[4 R^{2}-\left(r_{s}+r_{b}\right)^{2}\right] r^{2}}+\frac{\rho \pi^{2} N^{2}(3+\mu)}{28800} \cdot \frac{\left(R^{2}-r^{2}\right)\left[4 r^{2}-\left(r_{s}+r_{b}\right)^{2}\right]}{r^{2}} \\
\sigma_{\theta, H}=\frac{2 M\left(R^{2}+r^{2}\right)}{\pi f K\left(r_{b}-r_{s}\right)\left[4 R^{2}-\left(r_{s}+r_{b}\right)^{2}\right] r^{2}}+\frac{\rho \pi^{2} N^{2}(3+\mu)}{7200}\left[R^{2}+\frac{\left(R^{2}+r^{2}\right)\left(r_{s}+r_{b}\right)^{2}}{4 r^{2}}-\frac{1+3 \mu}{3+\mu} r^{2}\right] \\
\sigma_{r, S}=-\frac{2 M}{\pi f K\left(r_{b}-r_{s}\right)\left(r_{s}+r_{b}\right)^{2}}+\frac{\rho \pi^{2} N^{2}(3+\mu)}{28800}\left[\left(r_{s}+r_{b}\right)^{2}-4 r^{2}\right] \\
\sigma_{\theta, S}=-\frac{2 M}{\pi f K\left(r_{b}-r_{s}\right)\left(r_{s}+r_{b}\right)^{2}}+\frac{\rho \pi^{2} N^{2}(3+\mu)}{7200}\left[\frac{\left(r_{s}+r_{b}\right)^{2}}{4}-\frac{1+3 \mu}{3+\mu} r^{2}\right]
\end{gathered}
$$

\section{Numerical analysis}

This section studies the influence of the taper $K$ on the radial stresses and tangential stresses of the propeller hub and shaft. Extensive numerical computations have been performed with the following data:, Poisson's ratio $\mu_{C}=\mu_{S}=0.3$, the density $\rho_{C}=\rho_{S}=7800 \mathrm{~kg} / \mathrm{m}^{3}$, static friction coefficient $f=0.12$, the outer radius of the propeller hub $R=507.5 \mathrm{~mm}$, the radius of the small end of the propeller shaft $r_{s}=226 \mathrm{~mm}$, the radius of the big end of the propeller shaft $r_{b}=251.5 \mathrm{~mm}$. 


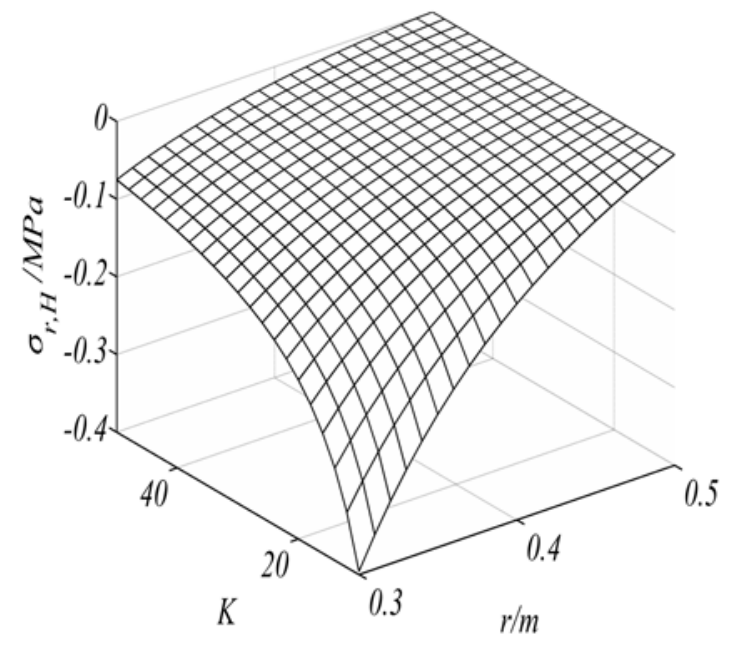

(a) Dependence of $\sigma_{r, H}$ upon $K$ for $N=50 \mathrm{r} / \mathrm{min}$.

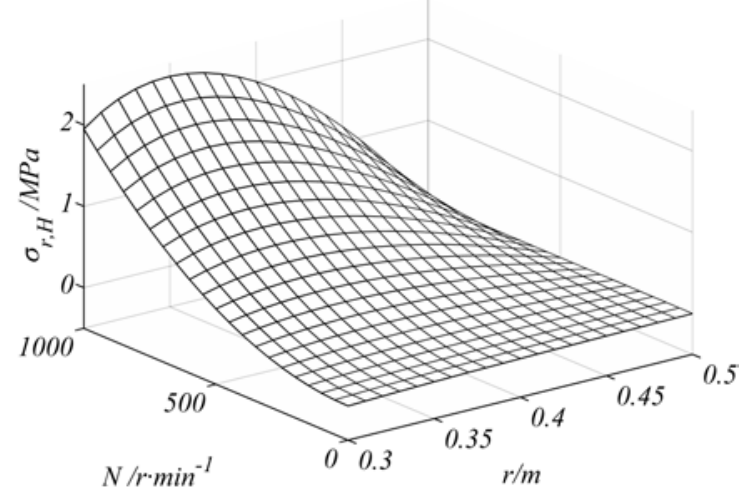

(b) Dependence of $\sigma_{r, H}$ upon $N$ for $K=50$.

Fig. 2 The radial stress of the hub.

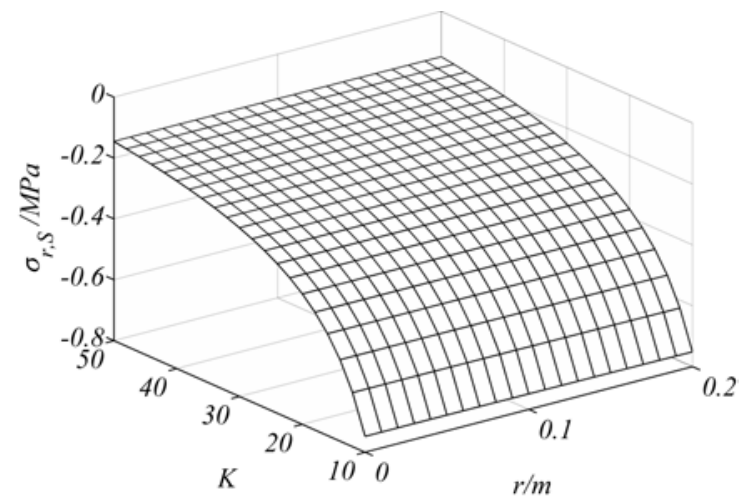


(a) Dependence of $\sigma_{r, s}$ upon $K$ for $N=50 \mathrm{r} / \mathrm{min}$.

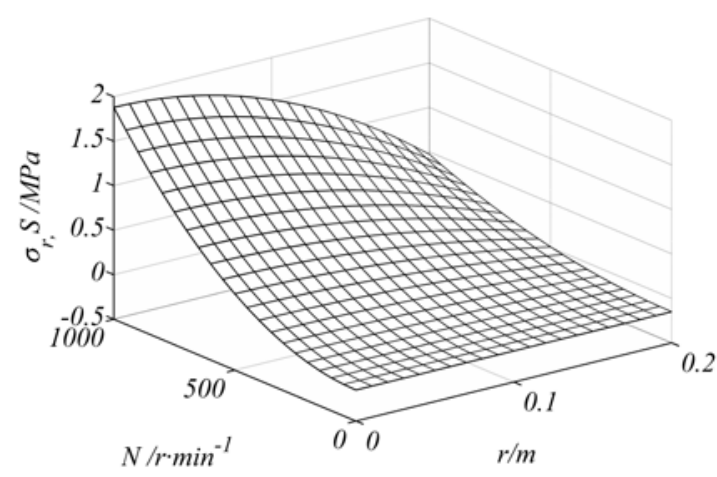

(b) Dependence of $\sigma_{r, S}$ upon $N$ for $K=50$.

Fig. 3 The radial stress of the shaft..

The influences of the taper and the rotating speed on the radial stresses of the propeller hub and shaft are shown in Fig. 2 and Fig. 3. $\sigma_{r, H}$ increases firstly as the radius increases, and then decreases as $r$ increases. The radial stress of the hub is reduced to zero for $r=0.5 \mathrm{~m}$. The maximum of $\sigma_{r, H}$ is closely related to the structure of the propeller hub. However, the growing of the radial stress of the shaft is insignificant as the radius $r$ increases. The maximum $\sigma_{r, S}$ happens in the center of the shaft.

In Fig. 4 and Fig. 5, the tangential stress of the propeller hub decrease with the growing of the radius. The dependence of the tangential stress upon the taper for the hub is similar as those for the propeller shaft. Namely, both of $\sigma_{\theta, H}$ and $\sigma_{\theta, S}$ are negatively correlated with the taper but positively correlated with the rotating speed.

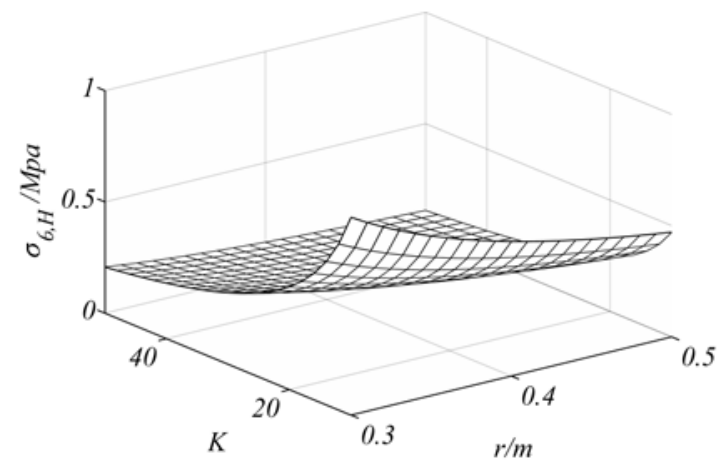

(a) Dependence of $\sigma_{\theta, H}$ upon $K$ for $N=50 \mathrm{r} / \mathrm{min}$. 


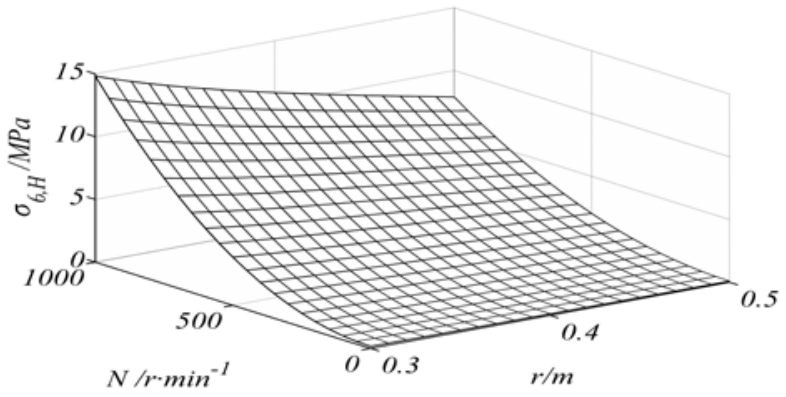

(b) Dependence of $\sigma_{\theta, H}$ upon $N$ for $K=50$.

Fig. 4 The tangential stress of the hub.

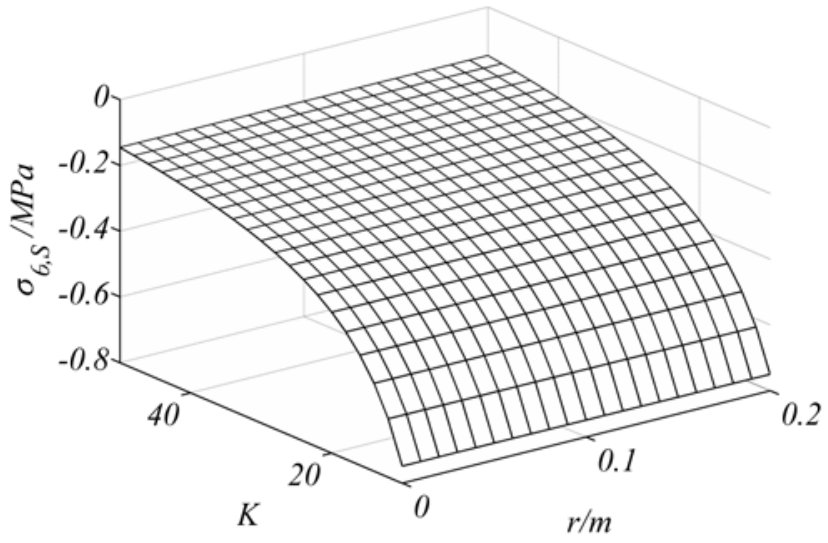

(a) Dependence of $\sigma_{\theta, S}$ upon $K$ for $N=50 \mathrm{r} / \mathrm{min}$.

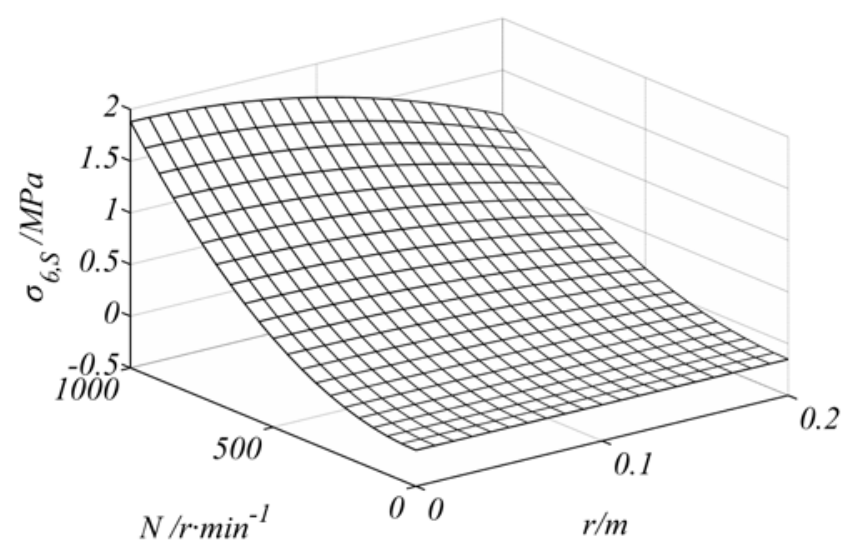

(b) Dependence of $\sigma_{\theta, S}$ upon $N$ for $K=50$.

Fig. 5 The tangential stress of the shaft. 


\section{Conclusions}

As a determine parameter, the taper have to be considered for the design of the tapered interference fit between the propeller hub and shaft. The maximum magnitudes of the stresses of the propeller hub are related to the structure of the screw propeller system.

The magnitudes of the radial stresses and tangential stresses of the propeller hub and the shaft are positively correlated with the rotating speed. Improving the stress distribution of the propeller hub is an effective approach to increase the connection strength and torque transmission capacity.

\section{References}

[1] F. G. Kollman: Journal of Mechanical Design Vol. 103 (1981), p. 61

[2] S.Sen and B.Aksakal: Materials and Design Vol. 25 (2004), p. 407-417

[3] D. Castagnetti and E. Dragoni: IMechE, J. Strain Analysis Vol. 40 (2005), p. $177-184$

[4] Peng Shang, Ying Zhan, Sheng Feng et al, Advanced Materials Research Vol. 744 (2013), p. 180-184

[5] M. M. Calistrat and G. G. Leaseburge, in: presented at the Amer. Soc. Mech. Eng. Conf. (1974).

[6] Seung Woo Lee and Dai Gil Lee: Composite Structures Vol. 78 (2007), p. 584-595

[7] Pierre-Daniel Pfister and Yves Perriard, in: Proc. 8th ICEMS, 3054-3059, Wuhan, China (2008)

[8] Guoping Wang, Hualing Chen, Shemiao Qi et al, in: 3rd International Conference on Machinery, Materials Science and Engineering Applications, Wuhan, China (2013)

[9] Ling Xiong, Peng Shang, Youlin Xu, Applied Mechanics and Materials Vol. 423 (2013), p. 1438-1443 